\title{
Filter Transients onboard DC Rolling Stock and Exploitation for the Estimate of the Line Impedance
}

\author{
Andrea Mariscotti \\ ASTM, Chiasso, Switzerland \\ andrea.mariscotti@astm-e.ch 0000-0002-0096-7305 \\ Antonio Delle Femine \\ University of Campania “L. Vanvitelli”, Aversa, Italy \\ antonio.dellefemine@unicampania.it 0000-0002-2503-8772
}

\author{
Domenico Giordano \\ INRIM, Torino, Italy \\ d.giordano@inrim.it 0000-0002-2616-9459 \\ Davide Signorino \\ INRIM, Torino, Italy \\ d.signorino@inrim.it 0000-0001-5141-6768
}

\begin{abstract}
Power supply transients in dc railways related to filter charging may trigger network and filter oscillatory responses, as well as cause very fast voltage spikes. These phenomena are relevant not only for Power Quality and EMC, but also for their impact on the measured pantograph quantities e.g. for power and energy consumption estimate. The broadband excitation of the system gives the possibility of attempting the identification of the network impedance. The experimental results are discussed and compared to the output of a circuit and a distributed parameter simulator. Matching between simulated and experimental data is very good.
\end{abstract}

Keywords-Harmonics, Power Quality, Power supply transient, Railways, Rolling stock

\section{INTRODUCTION}

Power Quality (PQ) in railways has been investigated from different standpoints: line voltage distortion coupled to other trains connected to the same line section or reflected back on the utility side [1]-[3]; current harmonics interfering with signaling and telecommunications [4]-[6]. Considering rolling stock and in a normative perspective, harmonics and distortion may affect the line voltage [7] or have impact also on the power and energy consumption assessment, both as an external unwanted interference [8] or as a contributing factor [9]. For DC vehicles harmonic active power was found to be negligible [9], although transients may disturb and alter the measured pantograph electrical quantities.

This work has as first objective the electrical characterization of the transients caused by the charging of the on-board filter of a dc locomotive. Transients for a dc vehicle originate at the sliding contact mechanism by electric arcs [12]-[14] or may be caused by the switching of onboard loads, including the case of a nearby train. Any electric transient with a broad frequency spectrum can excite the resonance modes of the system, thus triggering oscillations at a lower frequency, that can couple onto the measuring systems. Such oscillations and transients further worsen the stationarity of the signals associated to the line electrical quantities and weaken the conditions for the applicability of standardized Fourier-based processing methods [15].

The second objective is the investigation of the possibility

The results here presented are developed in the framework of the 16ENG04 MyRailS Project and the European Union's Horizon 2020 research and innovation program. The project received funding from the EMPIR programme co-financed by the Participating States and from the European Union's Horizon 2020 Research and Innovation Programme. This work was also supported by the Swiss State Secretariat for Education, Research and Innovation (SERI) under contract number 17.00127. The opinions expressed and arguments employed herein do not necessarily reflect the official views of the Swiss Government. of exploiting such transients as probing signals for a passive identification of the network impedance (i.e. the traction line impedance). The knowledge of the network impedance is beneficial for a more accurate estimate of harmonic distortion and the identification of disturbing loads, but also for network stability, harmonic resonance and prevention of oscillations [16][17].

The problem has already been considered for smart grids [18]-[21], using both active and passive methods: active methods inject a specific pilot signal to probe actively the network impedance; passive methods exploit existing measured quantities, passively listening to the network. A well-promising approach for passive methods is the use of advanced techniques for model identification (Least Mean Squares, recursive minimization, Kalman filter, etc.), but so far the adopted models are of the R-L type and neglect the effect of stray capacitance, with justifications that cannot be applied to railways and rapid transit systems.

The work is structured as follows: Section II contains an introduction to the problem of network modeling and network impedance identification, considering the approaches used in smart grids and distribution networks, and then focusing on railway networks and the interaction between the network and the locomotive; the results shown in Section III consist of the explanation of the observed electrical phenomena and interactions, and a preliminary estimate of the traction line impedance.

\section{NETWORK MODEL AND NETWORK IMPEDANCE ESTIMATE}

\section{A. General method}

The general method is based on the estimate of the load and/or network impedance exploiting two (or more) measurements of voltage and current at the pantograph (indicated with $V_{p}$ and $I_{p}$ ) carried out under different supply conditions. In other words the flowing current $I_{p}$ is measured after an ideally instantaneous change of the supply voltage. This method has been extensively applied to smart grids [18][22], with the following basic equations that assume a reference state and a perturbed state, e.g. by means of the inclusion/exclusion of a series-connected lumped element or ideally varying the network voltage supply.

The reference circuit is shown in Fig. 1, where the load (the train) is considered with its Norton equivalent circuit. The dashed resistance $R_{a}$ represents the switched lumped component suggested for application in distribution networks and smart grids, as well as the electric arc resistance, where electric arcs might be exploited as a driving event in a future development. 


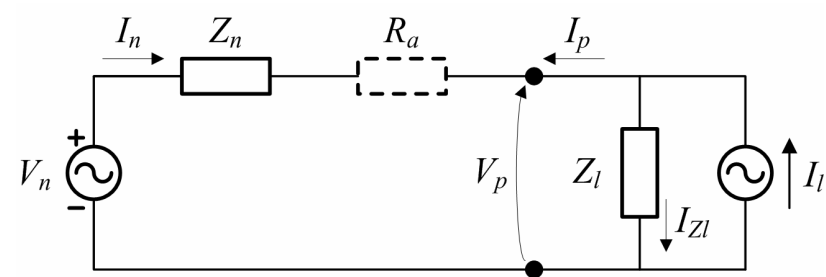

Fig. 1. Equivalent circuit for the supply network and rolling stock with electric arc occurring at the sliding contact (the resistance $R_{a}=0$ when the electric contact is perfect and no detachment occurs).

The basic equations are:

$$
I_{p}=I_{l}-I_{Z l} \quad I_{Z l}=\frac{V_{p}}{Z_{l}}
$$

The two equations must be written for each of the two states of the network, that is before and after the voltage change, with the quantities that vary identified with a prime and a double prime. Then replacing $I_{Z l}$ and solving for $Z_{l}$, and then focusing on $I_{l}$, it is possible to achieve two explicit expressions in terms of measured pantograph quantities only:

$$
Z_{l}=\frac{V_{p}^{\prime}-V_{p}^{\prime \prime}}{I_{p}^{\prime}-I_{p}^{\prime \prime}} \quad I_{l}=I_{p}^{\prime}+\frac{V_{p}^{\prime}}{Z_{l}}
$$

No similar closed-form expressions are available for the network impedance estimation, although in cases where the Norton impedance $Z_{l}$ is much larger than the network impedance $Z_{n}$, then $Z_{n}$ is readily given by $V_{p} / I_{p}$ for those frequencies at which the network generation $V_{n}$ may be passivated. The condition for passivation of the generator is that the network is not a source of significant voltage at a given frequency (e.g. choosing components different from the characteristic harmonics of the substation). Also in case of a moderately large $Z_{l}$, then the $Z_{n}$ can be calculated back from the electrical parallel of the two with an acceptable error. Considering $v_{p}(t)$ and $i_{p}(t)$, and the cross-spectrum $G_{u i}(f)$ and auto-spectrum $G_{i i}(f)$, a convenient way to express $Z_{n}$ is as the ratio of the two (holding the conditions on $Z_{l}$ ).

The Welch method is used for the calculation of the autospectrum and cross-spectrum.

\section{B. Other models}

As anticipated in the introduction, parametric methods make use of a reference model on which an optimization or identification algorith is run. In [20] the Kalman filter is a well founded approach, but the model is simplistic from two standpoints: the network is only resistive-inductive (RL series connection) and the selected state variables track only the fundamental and two harmonics (whereas we know that dc and ac railway systems feature complex spectra for the electrical quantities at the pantograph [23][24]).

The approach used in [19] makes direct use of the network model as in Fig. 1, relying on a redundant (overdetermined) set of equations ( 1 to $n$ ) to solve using an iterative technique. However, the main assumption is that the network is stationary, i.e. the configuration and generation do not change during the measurement.

Apparently a solution to the specific problem has been proposed very recently [25]. However, the focus is on an ac railway using an active probing method, i.e. injecting test signals by suitably driving the on-board four-quadrant converter as large-power waveform generator. This solution was already adopted for tests carried out with FLIRT trains with the permit of the operator and by modifying the software of the on-board converter (so, not an easy task), as documented in [26].

\section{Application to DC railways}

As a preliminary work on this topic, the approach is phenomenological, focusing on the electric behavior of the subsystems, the quality of the signals, and plausibility of the results. To this aim the signals are mostly studied in the time domain using a reference simulation model for confirmation. The measuring setup was preliminarily described in [27][28]: although the uncertainty assessment has not be completed yet, the used instrumentation and the care in grounding, shielding and EMC in general during the installation indicate that the uncertainty will be dominated by the voltage and current probes.

The simulation model must be fed with accurate estimates of the parameters to represent the real behavior of the line:

- it is known that using a few lumped parameters cells to model a distributed parameter system has a fitness error that increases with frequency and is particularly large at resonances and anti-resonances; stopping the reasoning to the first line resonance for a preliminary assessment allows the satisfactory use of a model with only two pi cells [5];

- lumped loads represented by the other trains in the same supply section or in the adjacent sections ca modify the line response, especially introducing additional damping at line resonances and in general slightly shifting line resonances due to the reactive elements onboard.

In the high-voltage input stage of the E464 locomotive shown in Fig. 2 the following elements can be recognized: the main circuit breaker (of the HSCB type, High-Speed Circuit Breaker, for dc applications), the second-order LC resonant filter (with the capacitance contributed mainly by the traction converters, but also at a lesser extent by the connected auxiliaries) and the charging system. The switchgear section is composed of the main circuit breaker and of an additional parallel-connected branch, with a contactor and the charging resistor $R_{c h}$ with a nominal value of $52 \Omega$.

Before closing the main HSCB supplying the locomotive, the input stage is configured to perform a pre-charge of the filter capacitors $C_{\text {filt_m }}$ (the capacitors inside the traction converters) and $C_{\text {filt_a }}$ (the capacitors inside the auxiliary converters) through the resistor $R_{c h}$. During the pre-charge the line current is absorbed from the catenary and delivered to the capacitors through the resistor $R_{c h}$ and the filter inductor $L_{\text {filt }}$ of $8.5 \mathrm{mH}$. After $0.8 \mathrm{~s}$, the voltage across the capacitor bank (made of the parallel-connected capacitors) reaches a given percentage of the line voltage, sufficient to conclude the charging operation more quickly through the main HSCB, closing it to by-pass the charging resistor. A smaller current impulse is then absorbed by the capacitor bank, where the limiting elements are the filter inductor $L_{\text {filt }}$ and the line impedance $Z_{p}$. The equivalent $R L C$ series circuit that during pre-charge was over-damped thanks to the $R_{c h}$ resistor becomes under-damped after the closing of the main HSCB, giving rise to visible oscillations (the only relevant resistive element contributing to damping being the supply line resistance). 


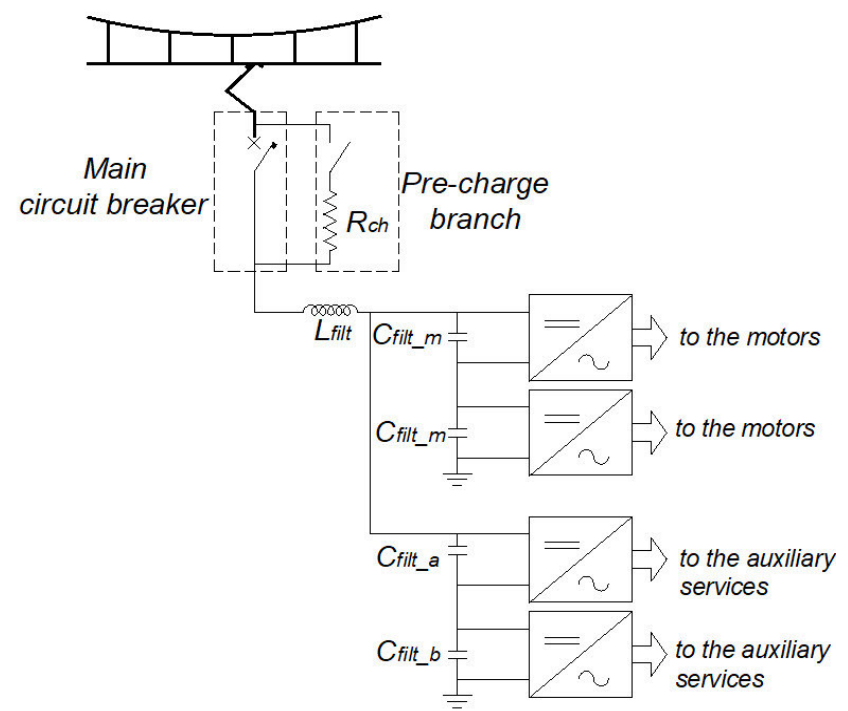

Fig. 2. Electrical scheme of the E464 locomotive, showing the onboard LC filter, the main circuit breaker and the pre-charging circuit with the limiting resistor $R_{c h}$

\section{RESULTS}

This section shows first the behavior of the filter charging transient associated to the circuit and procedure described in sec. II.C above (see Fig. 3). The observed electrical phenomena are explained, using the information of the supply and traction line models. The spectrum characteristics, the time duration, etc. are considered for their impact on Power Quality and on the connected measuring systems.

In the second part, then, portions of the measured waveforms are used to estimate the network impedance, verifying the quality of the so obtained estimate in terms of density of points on the frequency axis, noise and noise suppression capability, etc.

\section{A. Phenomenological analysis}

The two transients following the two switching events shown in Fig. 3 may be explained with a little insight into the behavior of the circuit. To start the charging of the filter capacitor, the pantograph is raised against the catenary using a series resistor $R_{c h}$ for protection purposes, limiting the inrush current into the capacitor bank through the filter inductor. The rate of rise of the current through the inductor is quite large anyway and excites the line resonance.

The steep edge of the current $I_{p}$ is visible in Fig. 3(b) lasting for about $80 \mu$ s and reaching a value of $32.6 \mathrm{~A}$. This corresponds to a $3800 \mathrm{~V}$ line voltage that charges an inductor of $8.5 \mathrm{mH}$, resulting in a rate of rise of $447 \mathrm{kA} / \mathrm{s}$, that after the said $80 \mu \mathrm{s}$ gives $36 \mathrm{~A}$. This first part of the rising exponential is able to excite the line resonance, resulting in a quick reduction of the pantograph voltage (the negative voltage spike); more accurately the tip of the spike occurs after $20 \mu \mathrm{s}$, when the current is less than $10 \mathrm{~A}$. It is briefly observed that a higher sampling rate is needed to better follow the evolution of the signal, when higher-order modes are excited: some recordings at a 5 or 10 times higher sampling rate are scheduled for the next test campaign.

The slower transient occurring when the charging resistor is bypassed at $2.34 \mathrm{~s}$ in Fig. 3(c) shows the damped oscillation of the onboard filter.
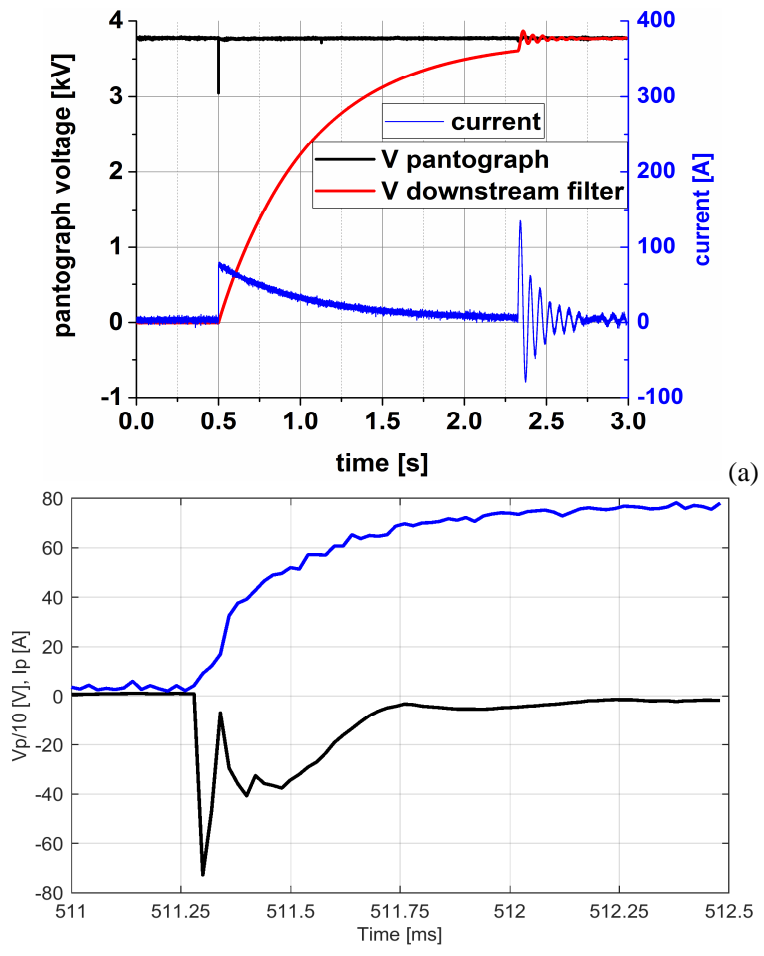

(b)

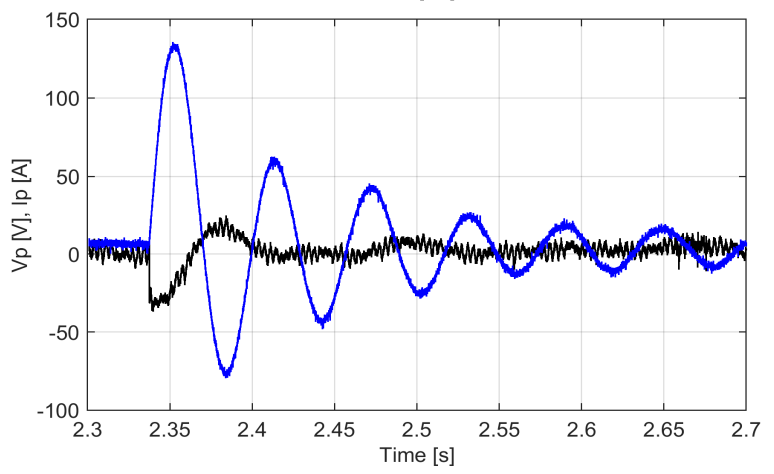

Fig. 3. Measured waveforms of pantograph voltage $V_{p}$, pantograph current $I_{p}$ and capacitor voltage downstream of filter $V_{f}$ : (a) overall screenshot of the relevant time interval, (b) zoom of the first insertion with resistor $R_{c h}$, (c) zoom of the residual oscillation when the resistor $R_{c h}$ is bypassed.

Two points should be observed:

- the current $I_{p}$ flowing in the filter is oscillating around zero; the negative portions of the current cannot in principle be absorbed back by the substation that consist of rectifier groups with all the diodes showing their cathodes facing the catenary; in reality, with a bias current flowing to supply other trains along the line, the diodes simply will see their current modulated by the oscillating charging current, with a dynamic resistance that is approximately constant if the charging current intensity is small compared to the current feeding the other trains (some other experimental results confirm that this situation may degenerate in low-traffic situations, when the supply section is not or lightly loaded);

- the oscillation has not a constant frequency: a closer look shows that the positive half-periods are slight but consistently longer than the negative ones (the measured intervals are $29.6,32.9$ and $32.1 \mathrm{~ms}$ for the positive halfperiods, and 28.8, 27.4 and $27.2 \mathrm{~ms}$ for the negative ones); this is a symptom of a non-linear characteristic that might be explained with the behavior of the substation diodes when the sourced current is very low. Variability was analyzed by parametric simulation, changing the load current biasing the substation diodes. 
The experimental curves of Fig. 3 allow also a preliminary evaluation of the impact caused by the transients triggered by the charging operation: the duration of the oscillation is in the order of $0.5 \mathrm{~s}$, its frequency is the resonant frequency of the onboard filter, usually between 15 and $22 \mathrm{~Hz}$ for the known implementations. The same phenomenon occurs whenever the traction demand is discontinued, e.g releasing the throttle during an intense acceleration, and it is a known problem for many out of scale readings of connected instruments.

The line resonance peak location and height depends on many factors, beside the geometrical and per-unit-length electrical parameters of the line: first of all the length of the line between the two adjacent substations and the presence of other trains in the same section, loading the line and reducing the factor of merit of the resonance. The curves of Fig. 4 show the variability of the network impedance at the pantograph $Z_{p}$ for a train moving along the line of $25 \mathrm{~km}$ length between two substations. The first and second resonances are located at $5.8 \mathrm{kHz}$ and $11.7 \mathrm{kHz}$ with a peak height between 400 and $4000 \Omega$ for the first and between 800 and $2000 \Omega$ for the second resonance. The height of the successive peaks is further reduced since the damping is quite large. The line voltage is quickly excited by the high-order resonances at the limit of the sampling time and beneath it $(50 \mathrm{kHz})$, then the profile in Fig. 3(b) around $511.5 \mathrm{~ms}$ shows a damped oscillation at a lower frequency, compatible with the first resonance. The negative spike reaches $-750 \mathrm{~V}$ and it is thus not dangerous in terms of overvoltage, but it is the byproduct of the broadband excitation of line resonances.

Observing the results in Fig. 5 the simulated current profile is quite well matched with the experimental one. However, the simulation model makes some approximations regarding the equivalent circuit for the substation rectifier and does not include a complete model for nearby trains, replaced by an equivalent loading resistor. The voltage profile is well matched for the initial high-frequency part, but the subsequent low-frequency damped oscillation needs some additional work.

\section{B. Identification of the network impedance}

The transient signals at the beginning of the charging operation (time $0.5 \mathrm{~s}$ in Fig. 3(b)) have been extracted for the calculation of the auto- and cross-spectrum. The procedure is complicated because of the limited number of samples (47) and the large amount of noise. The frequency resolution has been improved using interpolation; the noise is reduced by the averaging implicit in the Welch method.

The Welch method has unavoidably a lower frequency resolution than DFT, although the latter is a bad choice due to excessive spectral leakage, when processing signals with an underlying exponential damping component. In addition, spectrum estimation algorithms have in general better incoherent noise suppression than DFT.

The curve shown in Fig. 6 is affected by a large uncertainty due to the small number of original points, the averaging for the auto- and cross-spectrum estimates and the successive interpolation. However, it is very close to the one simulated in Fig. 4 for a line of $\mathrm{L}=25 \mathrm{~km}$ and train positioned at $\mathrm{x}=0.1 \mathrm{~L}(2.5 \mathrm{~km})$ from the substation (confirmed by the annotations during the tests).

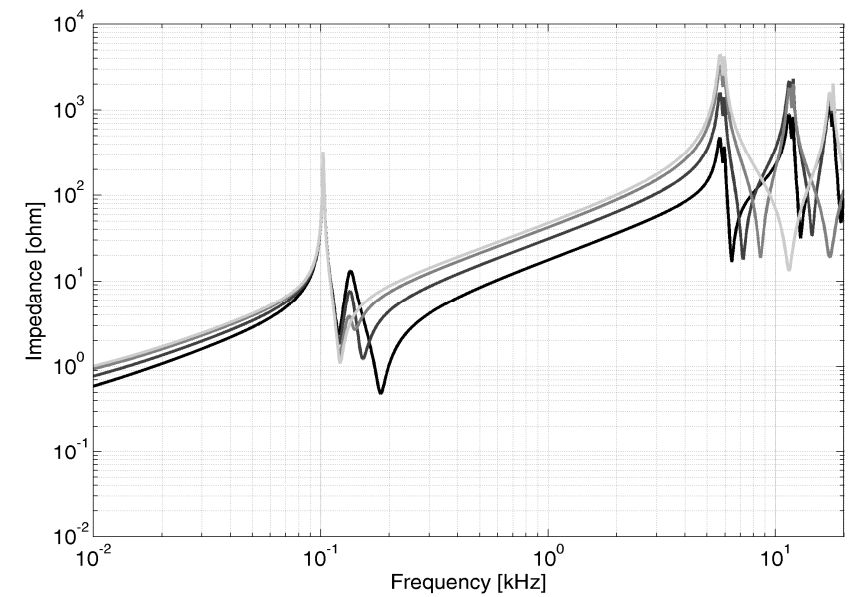

Fig. 4. Network impedance at the pantograph $\left(Z_{\mathrm{p}}\right)$ for $L=30 \mathrm{~km}$ of separation between ESSs and four train positions (from black to light grey) $\mathrm{x}=0.1,0.2,0.33,0.5 L[10]$.
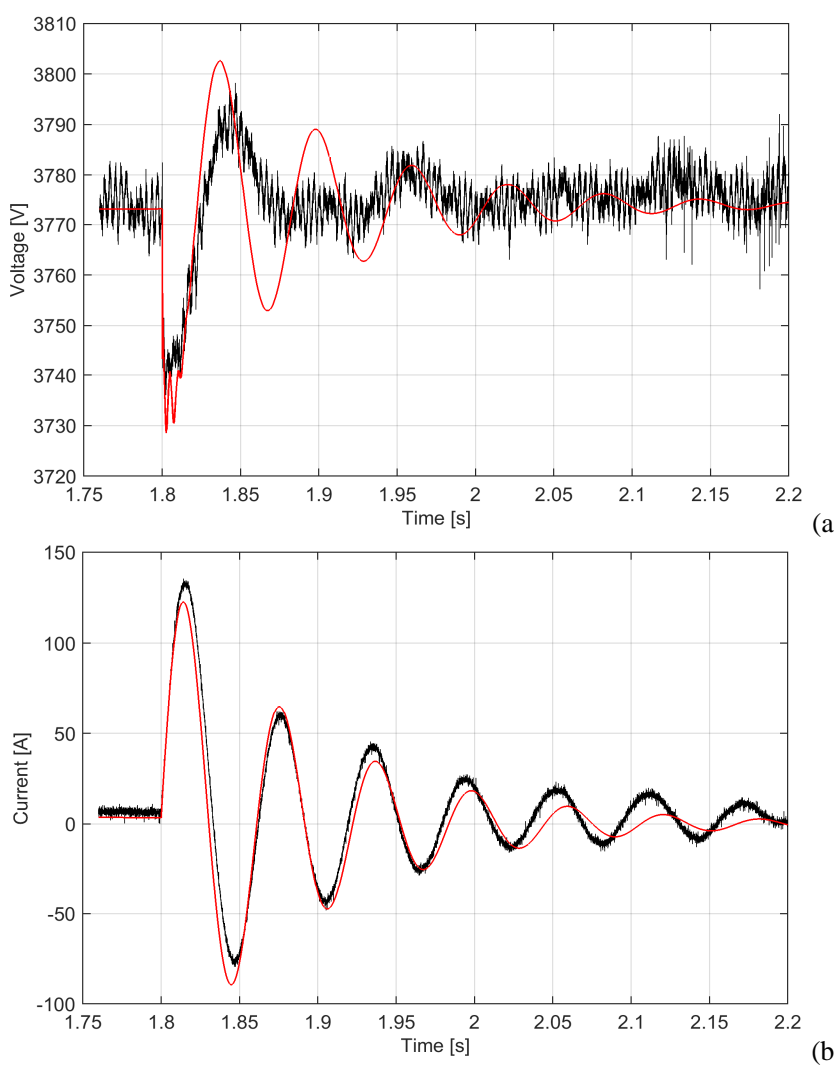

Fig. 5. Comparison of simulated (red) and measured (black) waveforms for (a) pantograph voltage $V_{p}$ and (b) pantograph current $I_{p}$ with fictitious line loading of $380 \mathrm{~A}$
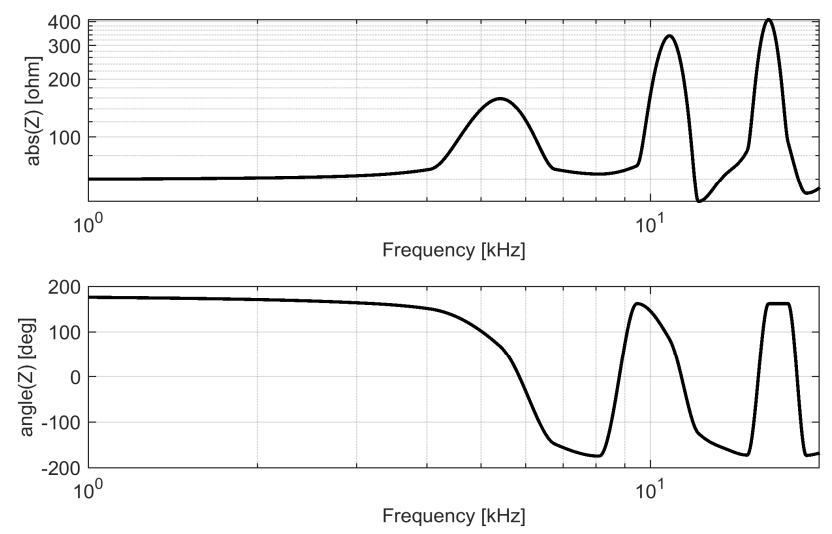

Fig. 6. Identified network impedance $Z_{n}$ after calculation of cross- and auto-spectrum, ratio, compensation of the electric parallel with the onboard filter, and interpolation to visually improve frequency resolution. 


\section{CONCLUSION}

This work has considered the problem of the identification of the network impedance for electrified railways, making a comparison to existing techniques for smart grids. It turns out that several methods have been implemented using simplified models of the network or simplifying assumptions, which hardly hold for railways and rapid transit systems: the pantograph impedance varies with the train movement and also with the relative position of the other trains. The problem is also complicated by the large amount of noise that characterizes the measured pantograph quantities.

For these reasons a parametric method should be considered, using a reference model and feeding data extracted from the measurements. Presently, although preliminarily considered, no numeric technique or automatic identification algorithm is used: data and results have been analyzed using a phenomenological approach, to understand what are the exploitable characteristics of the signals, the typical transient responses and the limitations of the adopted approach.

Network impedance estimation for railways (including dc and ac systems), besides the many challenges, may benefit from the abundance of electric transients caused by filter charging, transformer inrush, onboard auxiliaries' commutation, electric arcs. This work has focused on the onboard filter inrush for a dc locomotive.

The simulation results match well the experimental data, for what regards the filter oscillatory current, whereas there is some discrepancy in the calculated line voltage, possibly due to the non-linearity of the substation. The non-linearity of the system has been demonstrated by observing the asymmetry of the measured sinusoidal oscillation of the filter current.

The observations on the first transient when the pantograph is raised and the filter is supplied through the charging resistor are in agreement with the expected system response and the estimate of the spike in the line voltage quite accurately correspond to the measured values.

Last, the estimated network impedance match well the simulated curve obtained by the distributed parameter simulator, although the procedure and the uncertainty related to the many non idealities must be confirmed: the number of sampled data during the transient is too low and the Welch algorithm is thus operating in non-ideal conditions. To this aim the necessity of a higher sampling rate for the next test campaign has already been discussed.

\section{REFERENCES}

[1] J. Bongiorno, G. Boschetti and A. Mariscotti, "Low-Frequency Coupling: Phenomena in Electric Transportation Systems," IEEE Electrification Magazine, Vol. 4, n. 3, 2016, pp. 15-22.

[2] J. Holtz and H. J. Klein, "The propagation of harmonic currents generated by inverter fed locomotives in the distributed overhead supply systems," IEEE Trans. on Pow. Elect., Vol. 4, n. 2, Apr. 1989, pp. 168-174.

[3] M. Tanta, V. Monteiro, T. J. C. Sousa, A. P. Martins, A. S. Carvalho and J. L. Afonso, "Power quality phenomena in electrified railways: Conventional and new trends in power quality improvement toward public power systems," Proc. of the Intern. Young Engineers Forum, May 4, 2018, Lisbon, Portugal.

[4] Y. Li, C. H. Leow, W. L. Lau and M. C. K. Ong, "Induced voltage study and measurement for communication system in railway," Proc. of IEEE Intern. Symp. on Electrom. Comp., May 14, 2018, pp. 32-35.

[5] A. Mariscotti, M. Ruscelli and M. Vanti, "Modeling of Audiofrequency Track Circuits for validation, tuning and conducted interference prediction", IEEE Trans. on Intel. Transp. Sys., Vol. 11, n. 1, March 2010, pp. 52-60.

[6] G. Lucca, A. Bochicchio, M. Moro, "Electromagnetic interference on a telecommunication cable from a railway line: comparison between calculations and measurements," Proc. of 14th Intern. Zurich Symp. on Electromagnetic Compatibility, Feb. 20, 2001.

[7] CENELEC EN 50388, Railway Applications - Power supply and rolling stock - Technical criteria for the coordination between power supply (substation) and rolling stock to achieve interoperability, 2013.

[8] CENELEC EN 50463-2, Railway applications - Energy measurement on board trains, 2017.

[9] A. Mariscottti, "Characterization of Active Power Flow at Harmonics for AC and DC Railway Vehicles," IEEE Vehicle Power and Propulsion Conf., Oct. 14-17, 2019, Hanoi, Vietnam.

[10] A. Mariscotti and D. Giordano, "Electrical Characteristics of Pantograph Arcs in DC Railways: Infrastructure Influence," 23rd IMEKO TC4 Intern. Symp., Xi'an, China, Sept. 17-20, 2017.

[11] M. Meyer and J. Schöning, "Netzstabilität in großen Bahnnetzen," Eisenbahn-Revue, n. 7-8/1999, pp. 312-317.

[12] G. Crotti et al., "Pantograph-to-OHL Arc: Conducted Effects in DC Railway Supply System," IEEE Trans. on Instrum. and Meas., Vol. 68, n. 10, 2019, pp. 3861-3870.

[13] G. Gao, T. Zhang, W. Wei, Y. Hu, G. Wu and N. Zhou, "A pantograph arcing model for electrified railways with different speeds," Proc Inst. of Mech. Eng. - Part F: Journal of Rail and Rapid Transit, Vol. 32, n. 6, 2018, pp. 1731-1740.

[14] V. V. Terzija and H.-J. Koglin, "On the Modeling of Long Arc in Still Air and Arc Resistance Calculation," IEEE Trans. on Power Delivery, Vol. 19, n. 3, 2004, pp. 1012-1017.

[15] A. Mariscotti, "Characterization of Power Quality transient phenomena of DC railway traction supply", ACTA IMEKO, July 2012, Vol. 1, n. 1, pp. 26-35.

[16] H. Hu, H. Tao, F. Blaabjerg, X. Wang, Z. He and S. Gao, "TrainNetwork Interactions and Stability Evaluation in High-Speed Railways-Part I: Phenomena and Modeling," IEEE Trans. on Power Electronics, Vol. 33, n. 6, June 2018, pp. 4627-4642.

[17] H.J. Kaleybar, H.M. Kojabadi, M. Brenna, F. Foiadelli, S.S. Fazel and A. Rasi, "An Inclusive Study and Classification of Harmonic Phenomena in Electric Railway Systems," Proc. of IEEE Intern. Conf. on Envir. and Electr. Eng., June 11-14, 2019, Genova, Italy.

[18] E. Thunberg and L. Soder, "A Norton Approach to Distribution Network Modeling for Harmonic Studies," IEEE Trans. on Power Delivery, Vol. 14, n. 1, Jan. 1999, pp. 272-277.

[19] S. Cobreces, E. J. Bueno, D. Pizarro, F. J. Rodriguez and F. Huerta, "Grid Impedance Monitoring System for Distributed Power Generation Electronic Interfaces," IEEE Trans. on Instrum. and Meas., Vol. 58, n. 9, Sept. 2009, pp. 3112-3121.

[20] N. Hoffmann and F. W. Fuchs, "Minimal Invasive Equivalent Grid Impedance Estimation in Inductive-Resistive Power Networks Using Extended Kalman Filter," IEEE Trans. on Power Electronics, Vol. 29, n. 2, Feb. 2014, pp. 631-641.

[21] M. Ciobotaru, V. Agelidis and R. Teodorescu, "Line impedance estimation using model based identification technique," Proc. of the 14th European Conf. on Power Electronics and Applications, Aug. 30Sept. 1, 2011, Birmingham, UK.

[22] L. Asiminoaei, R. Teodorescu, F. Blaabjerg and U. Borup, "Implementation and Test of an Online Embedded Grid Impedance Estimation Technique for PV Inverters," IEEE Trans. on Industrial Electronics, Vol. 52, n. 4, Aug. 2005, pp. 1136-1144.

[23] A. Mariscotti, "Results on the Power Quality of French and Italian $2 \times 25 \mathrm{kV} 50 \mathrm{~Hz}$ railways", Proc. of Intern. Instrum. and Meas. Techn. Conf. I2MTC, Graz, Austria, May 13-16, 2012.

[24] A. Mariscotti, "DC railway line voltage ripple for periodic and aperiodic phenomena", Proc. of 18th IMEKO TC4 Symp. on Meas. of Electrical Quantities, Natal, RN, Brazil, Sept. 27-30, 2011.

[25] P. Pan, H. Hu, X. Yang, F. Blaabjerg, X. Wang and Z. He, "Impedance Measurement of Traction Network and Electric Train for Stability Analysis in High-Speed Railways," IEEE Trans. on Power Electronics, Vol. 33, n. 12, Dec. 2018, pp. 10086-10100.

[26] H.W.M. Smulders and H. A. Prins, "Fast and efficient determination of Eigenfrequencies of railway systems," Proc. of IEEE Intern. Instrum. and Meas. Techn. Conf. (I2MTC), May 11-14, 2015, Pisa, Italy.

[27] A. Delle Femine, D. Gallo, C. Landi and M. Luiso, "Discussion on DC and AC Power Quality Assessment in Railway Traction Supply Systems," Proc. of the IEEE Intern. Instrum. and Meas. Technology Conf., May 20-23, 2019, Auckland, New Zealand.

[28] G. Crotti et al., "Monitoring Energy and Power Quality On Board Train," Proc. of 10th IEEE Intern. Workshop on Applied Meas. for Power Systems, Sept. 25-27, 2019, Aachen, Germany. 